\title{
Number of existing permanent teeth is associated with chronic kidney disease in the elderly Korean population
}

\author{
Hye-Sun Shin
}

Department of Dental Hygiene, Eulji University College of Health Science, Seongnam, Korea

Received: November 24, 2016 Revised : January 20, 2017 Accepted: February 1, 2017

\section{Correspondence to} Hye-Sun Shin, Ph.D.

Department of Dental Hygiene, Eulji University College of Health Science, 553 Sanseong-daero, Sujeong-gu, Seongnam 13135, Korea

Tel: +82-31-740-7247

Fax: $+82-31-740-7352$

E-mail: bbanna82@snu.ac.kr
Background/Aims: The aim of this study was to assess the association between the number of existing permanent teeth and chronic kidney disease (CKD) in a representative sample of the elderly Korean population.

Methods: A total of 2,519 subjects who participated in the Korean National Health and Nutrition Examination Survey were cross-sectionally examined. The number of existing permanent teeth was evaluated by clinical oral examination. CKD was defined based on definition and classification by Kidney Disease: Improving Global Outcomes (KDIGO) 2012 guidelines. Multivariable logistic regression analyses were performed controlling for age, gender, income, education, tooth-brushing frequency, periodontitis, state of dentition, smoking, alcohol consumption, hypertension, obesity, diabetes mellitus, and hypercholesterolemia. Subgroup analyses by age and gender were also performed.

Results: The number of teeth was significantly associated with CKD after controlling for all potential confounders (adjusted odds ratio [AOR], 1.67; 95\% confidence interval [CI], 1.04 to 2.70 for lower number of teeth; AOR, 1.59; 95\% CI, 1.14 to 2.23 for moderate number of teeth). In the subgroup analyses, the association was highlighted in females aged 75 years over (AOR, 2.55; 95\% CI, 1.05 to 6.20 for lower number of teeth; AOR, 1.95; 95\% CI, 1.01 to 3.80 for moderate number of teeth).

Conclusions: Our findings suggest that the number of existing permanent teeth may be associated with CKD among Korean elderly.

Keywords: Renal insufficiency, chronic; Epidemiology; Number of teeth; Periodontitis; Risk factors

\section{INTRODUCTION}

Chronic kidney disease (CKD) is a grave public health issue with adverse outcomes such as hospitalization, kidney failure, cardiovascular diseases and premature mortality [1]. The prevalence of CKD is estimated to be greater than $10 \%$ worldwide [1]. According to the updated definition and criteria from the Kidney Disease:
Improving Global Outcomes (KDIGO) [2], CKD can be assessed through the estimated glomerular filtration rate (eGFR) and albuminuria stage. In Korea, the prevalence of CKD was reported to be $7.9 \%$ for 2011 to 2012, as determined by the Korean National Health and Nutrition Examination Survey (KNHANES) [3]. The higher risk of CKD showed a stronger association in females than males and in the older groups [3]. 
Periodontitis is a chronic inflammatory disease which leads to irreversible destruction of tissues of the teeth and surrounding structures [4]. It is closely related to several systemic diseases such as atherosclerosis [5] and metabolic syndromes [6]. Complicated outcomes such as severe periodontitis and untreated dental caries are the main causes of tooth loss [7], the prevalence of which greatly increases with age [8]. In addition, the predominance of adults aged 65 years and over with fewer than 19 natural teeth was reported to be approximately 50\% in Korea [9].

In a recent systematic review, there was evidence supporting the positive link between periodontitis and CKD and the beneficial effects of periodontal treatment on eGFR [10]. Two cohort [11,12] and 11 cross-sectional studies [13-23] have examined the association between periodontitis and CKD in the United States, Brazil, United Kingdom, Japan, China, and South Korea. Most presented positive results, except for two studies [14,17]. In addition, three reports showed that edentulous adults were significantly associated with CKD [14,20,24].

To date, no previous study has provided information on the association between CKD and the number of existing permanent teeth, which is usually related to oral diseases in the Korean elderly. Thus, we hypothesize that the number of permanent teeth is associated with CKD in the elderly Korean population.

This study aims to assess the association between the number of existing permanent teeth and CKD after considering various confounding variables such as age, gender, income, education, tooth-brushing frequency, periodontitis, state of dentition, smoking, alcohol consumption, hypertension, obesity, diabetes mellitus, and hypercholesterolemia. We also examined the strength of this association within different age and gender subgroups.

\section{METHODS}

\section{Study population}

The participants were drawn from the KNHANES collected by the Korea Center for Disease Control and Prevention (KCDC) in 2012 to 2014. The sampling protocol for the KNHANES was designed to include a complex, stratified, multistage, and probability-based sampling design with proportional allocation. The participants were randomly selected from diverse geographic areas, age, and gender groups from the 2005 National Census Registry. The survey consisted of questions regarding overall health, nutrition, and health examinations. The national survey was approved by the Institutional Review Board of the KCDC (2012-01EXP-01-2C, 2013-07CON03-4C, and 2013-12EXP-03-5C). All participants signed an informed consent form before participating in the study. The data for this research were acquired from household interviews, physical and oral examinations, and blood samples obtained from examination centers. Of the 23,626 subjects who participated in the KNHANES, exclusion criteria were as follows: $(1)<65$ years of age, (2) missing values in main variables and confounders such as oral examination, laboratory test for kidney function and various health variables. The final sample included 2,519 participants (1,101 males and 1,418 females).

\section{Assessment of chronic kidney disease}

The eGFR was calculated from serum creatinine levels using the Modification of Diet in Renal Disease (MDRD) equation: $\mathrm{eGFR}=175 \times$ serum creatinine $^{-1.154} \times$ age $^{-0.203} \times$ 0.742 (in females) [25]. The eGFR equation was validated for Asian populations and reported in $\mathrm{mL} / \mathrm{min} / 1.73 \mathrm{~m}^{2}$ [26]. eGFR were classified into five groups: eGFR $\geq 90$ $\mathrm{mL} / \mathrm{min} / 1.73 \mathrm{~m}^{2}(\mathrm{Gr}) ; \mathrm{e}$ GFR $=60$ to $89 \mathrm{~mL} / \mathrm{min} / 1.73 \mathrm{~m}^{2}$ (G2); eGFR = 45 to $59 \mathrm{~mL} / \mathrm{min} / 1.73 \mathrm{~m}^{2}$ (G3a); eGFR = 30 to $44 \mathrm{~mL} / \mathrm{min} / 1.73 \mathrm{~m}^{2}(\mathrm{G} 3 \mathrm{~b})$; and eGFR < $30 \mathrm{~mL} / \mathrm{min} / 1.73$ $\mathrm{m}^{2}\left(\mathrm{G}_{4}-5\right)$. Urinary albumin to creatinine ratios (UACRs) were calculated in milligrams per gram and divided into three categories by the KDIGO staging system [2]. UACR were classified into three groups: UACR $<30 \mathrm{mg} / \mathrm{g}\left(\mathrm{Al}_{1}\right)$; $\mathrm{UACR}=30$ to $300 \mathrm{mg} / \mathrm{g}\left(\mathrm{A}_{2}\right)$; and UACR $>300 \mathrm{mg} / \mathrm{g}\left(\mathrm{A}_{3}\right)$. $\mathrm{CKD}$ was defined using recent criteria that combined groups with eGFR $<60 \mathrm{~mL} / \mathrm{min} / 1.73 \mathrm{~m}^{2}$ and UACR $\geq$ $30 \mathrm{mg} / \mathrm{g}$ by the $2012 \mathrm{CKD}$ guideline released by KDIGO [2]. CKD was classified into four categories, those with no risk, moderate risks ( $\mathrm{G}_{3} \mathrm{a}-\mathrm{A}_{1}$ or $\left.\mathrm{G}_{1}-2 \mathrm{~A} 2\right)$, high risks (G3b-A1, G3a-A2, G1-2 $\left.A_{3}\right)$, and extremely high risks $\left(\mathrm{G}_{4}-5 \mathrm{~A}_{1}, \mathrm{G}_{3} \mathrm{~b}-5 \mathrm{~A}_{2}, \mathrm{G}_{3} \mathrm{a}-5 \mathrm{~A}_{3}\right)$ [2]. Finally, CKD was dichotomized with a yes or no parameter [27].

\section{Assessing the number of existing permanent teeth}

The number of existing permanent teeth was assessed by dentists, which excludes missing teeth, impacted 
teeth or implants and wisdom teeth. Root tips and teeth indicated for extraction were categorized as missing teeth. The number of existing permanent teeth was divided into three categories: o to 9,10 to 19 , and 20 to 28 . Han et al. $[28,29]$ recently reported that the cut-off for the number of teeth was 10 and 20 in the Korean population. The results of the oral health status also presented prevalence rates of the elderly who have 20 or more natural teeth through the KNHANES survey [9].

\section{Assessment of potential confounders}

Confounding variables used in statistical analyses included information on sociodemographic factors, oral health status and behaviors, and general health status and behaviors. Individual information from participants were gathered from personal interviews using structured questionnaires.

Household income was categorized into quartiles, while educational level was classified into four groups: less than primary school, middle school, high school, and more than college. The daily tooth-brushing frequency was categorized into two groups: less than twice a day and three or more times. Periodontal status and state of dentition were carefully assessed by dentists, in which the Community Periodontal Index of Treatment Needs (CPITN) was used to quantify periodontitis. The ten selected teeth were 11, 16, 17, 26, 27, 31, 36, 37, 46, and 47, according to the World Health Organization guidelines. Community periodontal index (CPI) was scored from o to 4: $\circ$ (normal), 1 (gingivitis with bleeding on probing), 2 (presence of calculus), 3 (pocket depth [PD] $\geq 3.5 \mathrm{~mm}$ ), and $4(\mathrm{PD} \geq 5.5 \mathrm{~mm})$. Periodontal status was grouped into two categories: absence (CPI 1 to 2 ) and presence (CPI 3 to 4 ) of periodontitis, while the state of dentition in the oral cavity was classified into three groups, namely natural dentition, removable partial denture and complete denture. Smoking status was categorized as never/former smokers and current smokers. The frequency of alcohol consumption was categorized into two groups: non-drinkers and drinkers (days per month or week). Hypertension was attributed to people with an average systolic blood pressure over $140 \mathrm{mmHg}$ or diastolic blood pressure over $90 \mathrm{mmHg}$ or medicated for hypertension. Body mass index was calculated as weight divided by the square of the height $\left(\mathrm{kg} / \mathrm{m}^{2}\right)$, and was classified into two groups: normal $\left(<25 \mathrm{~kg} / \mathrm{m}^{2}\right)$ and obese $\left(\geq 25 \mathrm{~kg} / \mathrm{m}^{2}\right)$. Diabetes mellitus was defined as having a fasting glucose level over $126 \mathrm{mg} / \mathrm{dL}$ or medicated for diabetes. Lastly, hypercholesterolemia was defined as having total cholesterol levels over $240 \mathrm{mg} / \mathrm{dL}$ or medicated for hypercholesterolemia.

\section{Statistical analyses}

Statistical analyses were performed following the KNHANES guidelines for applying complex survey designs and sampling weights. The outcome variable was CKD and the explanatory variable was the number of teeth. The characteristics of participants with or without CKD were presented using frequency distributions for the categorical variables with a chi-square test. All data were presented as weighted percentages and accompanied with calculations of standard errors. We presented the distribution of kidney function according to the number of teeth using a chi-square test. We then conducted logistic regression analyses to calculate crude odds ratio (OR) and adjusted odds ratio (AOR) with 95\% confidence intervals. Multivariable logistic regression analyses were sequentially applied to assess the association between the number of teeth and CKD after adjusting for age, gender, income, education, tooth-brushing frequency, periodontitis, state of dentition, smoking, alcohol consumption, hypertension, obesity, diabetes mellitus, and hypercholesterolemia. Model 1 represents a crude association, while model 2 is adjusted for sociodemographic factors. Model 3 is adjusted for all the variables in model 2, including oral health status and behaviors, and lastly model 4 is adjusted for all the variables in model 3, including general health status and behaviors. We evaluated the fully AOR while adjusting for confounding and other variables. Subgroup analyses by age and gender were also performed to identify risk groups. All analyses were performed using the SPSS version 19.0 (IBM Co., Armonk, NY, USA).

\section{RESULTS}

\section{Characteristics of the study participants}

Of the 2,519 participants included in the analyses, 578 (22.9\%) were classified with CKD (Table 1). The univariate association between the explanatory and confounding variables and CKD was examined using chi-square 
Shin HS. The number of teeth and kidney disease

Table 1. Characteristics of the participants according to chronic kidney disease $(n=2,519)$

\begin{tabular}{|c|c|c|c|c|}
\hline Characteristic & Number & Non-CKD $(n=1,941)$ & $\mathrm{CKD}(\mathrm{n}=578)$ & $p$ value $^{\mathrm{b}}$ \\
\hline Age, yr & & & & $<0.001^{\mathrm{c}}$ \\
\hline $65-74$ & 1,723 & $1,402(71.6)^{\mathrm{a}}$ & $321(54.8)^{\mathrm{a}}$ & \\
\hline$\geq 75$ & 796 & $539(27.4)$ & $257(45.2)$ & \\
\hline Sex & & & & 0.343 \\
\hline Male & 1,101 & $835(43.1)$ & $266(45.8)$ & \\
\hline Female & 1,418 & $1,106(56.9)$ & $312(54.2)$ & \\
\hline Monthly Household income & & & & 0.902 \\
\hline Lowest quartile & 579 & $442(24.2)$ & $137(22.5)$ & \\
\hline Lower middle quartile & 623 & $476(25.8)$ & $147(26.5)$ & \\
\hline Upper middle quartile & 643 & $495(24.8)$ & $148(25 \cdot 3)$ & \\
\hline Highest quartile & 674 & $528(25.2)$ & $146(25 \cdot 7)$ & \\
\hline Education & & & & 0.445 \\
\hline Primary school & 1,496 & $1,154(60.9)$ & $342(62.1)$ & \\
\hline Middle school & 355 & $270(14.5)$ & $85(13.8)$ & \\
\hline High school & 434 & $327(15.8)$ & $107(17.2)$ & \\
\hline College & 234 & $1,190(8.8)$ & $44(6.8)$ & \\
\hline Tooth-brushing frequencies & & & & 0.416 \\
\hline$<2$ & 481 & $362(19.7)$ & $119(21.5)$ & \\
\hline$\geq 2$ & 2,038 & $1,579(80.3)$ & $459(78.5)$ & \\
\hline Periodontitis & & & & 0.499 \\
\hline No & 1,395 & $1,065(53.8)$ & $330(55 \cdot 7)$ & \\
\hline Yes & 1,124 & $876(46.2)$ & $248(44 \cdot 3)$ & \\
\hline State of dentition & & & & 0.346 \\
\hline Natural dentition & 1,642 & $1,282(64.5)$ & $360(61.4)$ & \\
\hline $\mathrm{RPD}$ & 606 & $452(24 \cdot 3)$ & $154(27.7)$ & \\
\hline $\mathrm{CD}$ & 271 & $207(11.2)$ & $64(10.9)$ & \\
\hline Smoking status & & & & 0.066 \\
\hline Never/former & 2,240 & $1,739(89.9)$ & $501(86.6)$ & \\
\hline Current & 279 & $202(10.1)$ & $77(13.4)$ & \\
\hline Alcohol consumption & & & & 0.186 \\
\hline No & 1,153 & $866(45.0)$ & $287(48.4)$ & \\
\hline Yes & 1,366 & $1,075(55.0)$ & $291(51.6)$ & \\
\hline Hypertension & & & & $<0.001^{\mathrm{c}}$ \\
\hline No & 1,025 & $885(45.9)$ & $140(24.6)$ & \\
\hline Yes & 1,494 & $1,056(54.1)$ & $438(75.4)$ & \\
\hline Obesity & & & & $0.001^{\mathrm{c}}$ \\
\hline No & 1,633 & $1,298(66.1)$ & $335(57 \cdot 3)$ & \\
\hline Yes & 886 & $643(33.9)$ & $243(42.7)$ & \\
\hline Diabetes mellitus & & & & $<0.001^{\mathrm{c}}$ \\
\hline No & 1,981 & $1,607(81.6)$ & $374(65.0)$ & \\
\hline Yes & 538 & $334(18.4)$ & $204(35 \cdot 0)$ & \\
\hline Hypercholesterolemia & & & & 0.242 \\
\hline No & 1,865 & $1,445(74.9)$ & $420(72.2)$ & \\
\hline Yes & 654 & $496(25.1)$ & $158(27.8)$ & \\
\hline The number of existing pern & & & & $0.002^{c}$ \\
\hline Low $(0-9)$ & 364 & $266(14.0)$ & $98(16.3)$ & \\
\hline Moderate (10-19) & 643 & $469(25.4)$ & $174(32.7)$ & \\
\hline $\operatorname{High}(20-28)$ & 1,512 & $1,206(60.6)$ & $306(51.1)$ & \\
\hline
\end{tabular}

Values are presented as number (\%).

CKD, chronic kidney disease; RPD, removal partial denture; CD, complete denture.

${ }^{a}$ Weighted percentage was calculated based on the complex sample design and weights.

${ }^{b}$ Obtained from chi-square test.

${ }^{\text {c}}$ Statistical significance at $p<0.05$. 
Table 2. Distribution of kidney function according to the number of existing permanent teeth $(n=2,519)$

\begin{tabular}{|c|c|c|c|c|c|}
\hline \multirow{2}{*}{ Variable } & \multirow{2}{*}{ Number } & \multicolumn{3}{|c|}{ The number of existing permanent teeth } & \multirow{2}{*}{$p$ value $^{\mathrm{a}}$} \\
\hline & & $0-9(n=364)$ & $10-19(n=643)$ & $20-28(n=1,512)$ & \\
\hline \multicolumn{6}{|l|}{ eGFR stage } \\
\hline $\mathrm{G} 1\left(\geq 90 \mathrm{~mL} / \mathrm{min} / 1.73 \mathrm{~m}^{2}\right)$ & 582 & $19.2(2.4)$ & $24 \cdot 3(2.0)$ & $23.9(1.5)$ & $0.024^{b}$ \\
\hline $\mathrm{G} 2\left(60-89 \mathrm{~mL} / \mathrm{min} / 1.73 \mathrm{~m}^{2}\right)$ & 1,656 & $65.7(2.8)$ & $65.2(2.3)$ & $66.2(1.5)$ & \\
\hline $\mathrm{G}_{3 \mathrm{a}}\left(45-59 \mathrm{~mL} / \mathrm{min} / 1.73 \mathrm{~m}^{2}\right)$ & 231 & $10.8(1.8)$ & $9.0(1.2)$ & $8.6(0.9)$ & \\
\hline $\mathrm{G}_{3} \mathrm{~b}\left(30-44 \mathrm{~mL} / \mathrm{min} / 1.73 \mathrm{~m}^{2}\right)$ & 40 & $3.0(1.0)$ & $1.3(0.4)$ & $1.1(0.3)$ & \\
\hline $\mathrm{G}_{4}-5\left(<30 \mathrm{~mL} / \mathrm{min} / 1.73 \mathrm{~m}^{2}\right)$ & 10 & $1.3(0.6)$ & $0.2(0.2)$ & $0.2(0.1)$ & \\
\hline \multicolumn{6}{|l|}{ UACR } \\
\hline $\mathrm{Al}(<30 \mathrm{mg} / \mathrm{g})$ & 2,139 & $82.9(2.2)$ & $79.4(2.1)$ & $86.5(1.1)$ & $0.011^{\mathrm{b}}$ \\
\hline $\mathrm{A} 2(30 \leq \mathrm{UCAR} \leq 300 \mathrm{mg} / \mathrm{g})$ & 337 & $15 \cdot 5(2.1)$ & $18.1(2.0)$ & $12.3(1.0)$ & \\
\hline $\mathrm{A}_{3}(>300 \mathrm{mg} / \mathrm{g})$ & 43 & $1.5(0.8)$ & $22.5(0.8)$ & $1.2(0.3)$ & \\
\hline \multicolumn{6}{|l|}{ CKD category } \\
\hline No CKD & 1,941 & $73.7(2.5)$ & $71.7(2.3)$ & $79.5(1.3)$ & $0.001^{b}$ \\
\hline Moderate risk & 443 & $17.7(2.2)$ & $22.5(2.2)$ & $16.5(1.2)$ & \\
\hline High risk & 100 & $5 \cdot 7(1.3)$ & $4.9(1.0)$ & $3.2(0.5)$ & \\
\hline Very high risk & 35 & $3.0(1.0)$ & $1.0(0.4)$ & $0.9(0.2)$ & \\
\hline \multicolumn{6}{|l|}{ CKD } \\
\hline No & 1,941 & $73.7(2.5)$ & $71.7(2.3)$ & $79.5(1.3)$ & $0.002^{b}$ \\
\hline Yes & 578 & $26.3(2.5)$ & $28.3(2.3)$ & $20.5(1.3)$ & \\
\hline
\end{tabular}

Values are presented as number (\%).

eGFR, estimated glomerular filtration; UACR, urinary albumin to creatinine ratio; CKD, chronic kidney disease.

${ }^{\mathrm{a}}$ Obtained from chi-square test.

${ }^{\mathrm{b}}$ Statistical significance at $p<0.05$.

tests. The percentage of participants with CKD was significantly higher in the 65- to 74-year-old age group. Hypertension, obesity, and diabetes mellitus were significantly different between CKD and non-CKD groups.

\section{Distribution of CKD as a function of teeth number}

Table 2 shows the distribution of kidney function according to the number of teeth. Participants with fewer teeth had significantly lower eGFR and higher UACR. Consequently, the CKD category was presented with decreased eGFR and increased UACR. Participants with fewer number of teeth were more likely to suffer from a $\mathrm{CKD}$ risk when compared to participants with a greater number of teeth.

\section{Association between the number of teeth and CKD}

Through a crude analysis, the number of teeth was found to be associated with CKD (Table 3). After adjusting for sociodemographic factors, the association between low- er number of teeth and CKD disappeared for model 2. In models 3 and 4 , the strength of the association between the number of teeth and CKD remained significant. A dose-effect trend was observed for the association.

\section{Association between CKD and potential confound- ing variables}

We evaluated the AOR of each confounder through a fully adjusted model (Table 4). Confounding variables included participants aged 75 and older, who are currently smoking, hypertension, obesity, and diabetes mellitus associated with CKD. Periodontitis and complete denture were negatively associated with CKD.

\section{Age- and gender-stratified association between the number of teeth and CKD}

The stratified association between the number of teeth and CKD was highlighted in the specific subgroups (Table 5). In analyses in which age and gender were both tak- 
Table 3. Adjusted association between the number of existing permanent teeth and chronic kidney disease $(n=2,519)$

\begin{tabular}{|c|c|c|c|c|c|}
\hline \multirow{2}{*}{ Variable } & \multirow{2}{*}{ Number } & \multicolumn{4}{|c|}{ OR (95\% CI) } \\
\hline & & Model $1^{a}$ & Model $2^{\mathrm{b}}$ & Model $3^{c}$ & Model $4^{\mathrm{d}}$ \\
\hline \multicolumn{6}{|c|}{ The number of existing permanent teeth } \\
\hline $0-9$ & 364 & $1.38(1.04-1.83)^{\mathrm{e}}$ & $1.16(0.86-1.57)$ & $1.69(1.08-2.64)^{\mathrm{e}}$ & $1.67(1.04-2.70)^{\mathrm{e}}$ \\
\hline $10-19$ & 643 & $1.53(1.18-1.98)^{\mathrm{e}}$ & $1.41(1.08-1.83)^{\mathrm{e}}$ & $1.65(1.20-2.28)^{\mathrm{e}}$ & $1.59(1.14-2.23)^{\mathrm{e}}$ \\
\hline $20-28$ & 1,512 & 1 & 1 & 1 & 1 \\
\hline
\end{tabular}

The dependent variable was chronic kidney disease.

OR, odds ratio; CI, confidence interval.

${ }^{\mathrm{a}}$ Model 1 was unadjusted association.

${ }^{\mathrm{b}}$ Model 2 was adjusted for age, gender, income, and education.

${ }^{\mathrm{c}}$ Model 3 was adjusted for all variables in model 2 and tooth-brushing frequencies, periodonititis, and state of dentition.

${ }^{\mathrm{d}}$ Model 4 was adjusted for all variables in model 3 and smoking, alcohol consumption, hypertension, obesity, diabetes mellitus, and hypercholesterolemia.

${ }^{\mathrm{e}}$ Statistical significance at $\mathrm{p}<0.05$.

en into consideration, the strength of the link between the number of teeth and CKD specifically increased in females aged 75 years and over.

\section{DISCUSSION}

To our knowledge, the findings of this study provide the evidence associating the number of existing permanent teeth and CKD, independent of traditional risk factors such as hypertension, diabetes mellitus, smoking, and various potential confounders.

Although a growing body of evidence have demonstrated an association between periodontitis, edentulism and CKD, the concordance remains controversial [10-23]. Analyses of population-based data from 12,947 participants showed a positive association between edentulism and periodontal disease on CKD in the United States population(OR, 1.60 for periodontitis; OR, 1.85 for edentulism) [15]. Another study presented a positive association between only edentulism and CKD in the National Health and Nutrition Examination Survey (OR, 1.64) [14]. Others have emphasized that individuals with CKD have to recognize the impact of periodontitis on the disease, and the importance of periodontal treatment [30]. Through a United States national survey, periodontitis, and edentulism were identified to potentially increase the mortality of participants with CKD [31]. They suggested that periodontal disease may be a crucial risk factor for cardiovascular diseases and mortality in general for participants with CKD. In contrast, periodontitis is inversely associated with CKD in our study. Although there is only one existing study on the association between periodontitis and CKD in the Korean population [23], the results were contradictory with the present study. The conflicting results may be influenced by age distribution, definition, and criteria of $\mathrm{CKD}$, and confounding variables. Additional studies will be needed to confirm the link between periodontitis and CKD in the Korean population.

The results of this study indicated that lower numbers of teeth had significantly higher AOR than moderate numbers in a fully adjusted model. Our data cannot compare with other studies due to the absence of information linking the number of teeth and CKD. In our subgroup analyses, the link between the number of teeth and CKD showed a stronger association in females aged 75 years and over when compared to the total participants (AOR, 2.55 for lower number of teeth; AOR, 1.95 for moderate number of teeth). We then speculated potential arguments for these results. According to previous findings, age and gender differences exist in the prevalence of tooth loss [9] and CKD [3]. The higher risk for CKD and severe tooth loss showed a stronger association in females than in males, and in the older age group than the younger [3,9]. Therefore, the association between CKD and tooth loss may play a more significant role in females aged 75 years and over than any other group. The causal relationship between the number of teeth and CKD has yet to be established. 
Table 4. Adjusted odds ratio for chronic kidney disease $(n=2,519)$

\begin{tabular}{|c|c|c|}
\hline Variable & Number & Fully adjusted $\mathrm{OR}^{\mathrm{a}}(95 \% \mathrm{CI})$ \\
\hline \multicolumn{3}{|l|}{ Age, yr } \\
\hline $65-74$ & 1,723 & 1 \\
\hline$\geq 75$ & 796 & $1.99(1.57-2.52)^{b}$ \\
\hline \multicolumn{3}{|l|}{ Sex } \\
\hline Male & 1,101 & $1.30(0.99-1.72)$ \\
\hline Female & 1,418 & 1 \\
\hline \multicolumn{3}{|l|}{ Monthly Household income } \\
\hline Lowest quartile & 579 & $0.89(0.64-1.23)$ \\
\hline Lower middle quartile & 623 & $1.05(0.74-1.49)$ \\
\hline Upper middle quartile & 643 & $1.01(0.74-1.38)$ \\
\hline Highest quartile & 674 & 1 \\
\hline \multicolumn{3}{|l|}{ Education } \\
\hline Primary school & 1,496 & $1.21(0.76-1.93)$ \\
\hline Middle school & 355 & $1.19(0.74-1.91)$ \\
\hline High school & 434 & $1.31(0.84-2.03)$ \\
\hline College & 234 & 1 \\
\hline \multicolumn{3}{|c|}{ Tooth-brushing frequencies } \\
\hline$<2$ & 481 & $1.01(0.75-1.36)$ \\
\hline$\geq 2$ & 2,038 & 1 \\
\hline \multicolumn{3}{|l|}{ Periodontitis } \\
\hline No & 1,395 & 1 \\
\hline Yes & 1,124 & $0.78(0.62-0.98)^{b}$ \\
\hline \multicolumn{3}{|l|}{ State of dentition } \\
\hline Natural dentition & 1,642 & 1 \\
\hline $\mathrm{RPD}$ & 606 & $0.74(0.52-1.05)$ \\
\hline $\mathrm{CD}$ & 271 & $0.53(0.31-0.92)^{b}$ \\
\hline \multicolumn{3}{|l|}{ Smoking status } \\
\hline Never/former & 2,240 & 1 \\
\hline Current & 279 & $1.58(1.07-2.32)^{b}$ \\
\hline \multicolumn{3}{|l|}{ Alcohol consumption } \\
\hline No & 1,153 & 1 \\
\hline Yes & 1,366 & $0.84(0.66-1.07)$ \\
\hline \multicolumn{3}{|l|}{ Hypertension } \\
\hline No & 1,025 & 1 \\
\hline Yes & 1,494 & $2.26(1.74-2.93)^{b}$ \\
\hline \multicolumn{3}{|l|}{ Obesity } \\
\hline No & 1,633 & 1 \\
\hline Yes & 886 & $1.39(1.09-1.78)^{b}$ \\
\hline \multicolumn{3}{|l|}{ Diabetes mellitus } \\
\hline No & 1,981 & 1 \\
\hline Yes & 538 & $2.14(1.63-2.81)^{b}$ \\
\hline \multicolumn{3}{|l|}{ Hypercholesterolemia } \\
\hline No & 1,865 & 1 \\
\hline Yes & 654 & $1.04(0.79-1.36)$ \\
\hline
\end{tabular}

The dependent variable was chronic kidney disease.

OR, odds ratio; CI, confidence interval; RPD, removal partial denture; CD, complete denture.

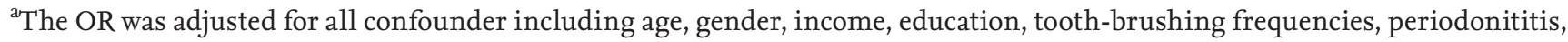
state of dentition, smoking, alcohol consumption, hypertension, obesity, diabetes mellitus, and hypercholesterolemia.

${ }^{\mathrm{b}}$ Statistical significance at $p<0.05$. 
Table 5. Age- and gender- stratified adjusted association of the number of existing permanent teeth and chronic kidney disease $(n=2,519)$

\begin{tabular}{|c|c|c|c|c|}
\hline \multirow{2}{*}{ Stratum } & \multirow{2}{*}{ Number } & \multicolumn{3}{|c|}{ Adjusted OR (95\% CI) } \\
\hline & & $0-9$ & $10-19$ & $20-28$ \\
\hline \multicolumn{5}{|l|}{ Age, yr } \\
\hline $65-74$ & 1,723 & $1.44(0.71-2.93)$ & $1.52(0.98-2.35)$ & 1 \\
\hline$\geq 75$ & 796 & $1.97(0.98-3.96)$ & $1.70(1.05-2.75)^{\mathrm{a}}$ & 1 \\
\hline \multicolumn{5}{|l|}{ Sex } \\
\hline Male & 1,101 & $1.48(0.71-3.08)$ & $1.71(1.02-2.85)^{\mathrm{a}}$ & 1 \\
\hline Female & 1,418 & $2.01(1.04-3.87)^{\mathrm{a}}$ & $1.54(1.01-2.34)^{\mathrm{a}}$ & 1 \\
\hline \multicolumn{5}{|l|}{ Age and sex } \\
\hline 65- to 74-year-old male & 745 & $1.33(0.47-3.81)$ & $2.16(1.12-4.17)^{\mathrm{a}}$ & 1 \\
\hline 65- to 74-year-old female & 978 & $1.76(0.65-4.78)$ & $1.17(0.66-2.07)$ & 1 \\
\hline z 75-year-old male & 356 & $1.62(0.59-4.49)$ & $1.26(0.62-2.56)$ & 1 \\
\hline$\geq 75$-year-old female & 440 & $2.55(1.05-6.20)^{\mathrm{a}}$ & $1.95(1.01-3.80)^{\mathrm{a}}$ & 1 \\
\hline
\end{tabular}

The dependent variable was chronic kidney disease. Models were adjusted for age, gender, income, education, tooth-brushing frequencies, periodontitis, state of dentition, smoking, alcohol consumption, hypertension, obesity, diabetes mellitus, and hypercholesterolemia except the stratum. OR, odds ratio; CI, confidence interval.

${ }^{a}$ Statistical significance at $p<0.05$.

The biological reasoning linking tooth loss to decreased kidney function may be explained by a few related theories. Chewing difficulties due to tooth loss may affect food diversity and dietary patterns [32], which can lead to poor nutrition [33]. Nutritional deficiencies may cause weight loss and development of major chronic diseases and cardiovascular diseases [34]. Moreover, dietary patterns are associated with cardiometabolic and endocrine biomarkers. Previous studies demonstrate that the dietary modification were effective in controlling blood pressure and diabetes [35,36]. Chronic diseases such as diabetes mellitus and hypertension are traditional risk factors for the negative consequences associated with CKD. Thus, our study suggests that tooth loss can affect CKD through hypertension and diabetes mellitus, which are important risk factors for CKD.

In this study, the number of existing permanent teeth is an indicator of oral health status, which is primarily examined among elderly populations in oral health research. Kressin et al. [37] reported that tooth-brushing, dental floss use, regular prophylaxis, and combinations of oral health-related behaviors can lead to tooth retention. Our data suggest that managing and maintaining oral health is important throughout life, and that the number of teeth may affect CKD at old age.

That said, there are some limitations to this study. First, since there were no previous studies of the same nature, we could not make a detailed comparison of our findings, and thus additional evidence are needed to clarify the association. Recent studies have reported the impact of tooth loss on dietary intake in individuals with CKD [38], in which nutritional interventions may contribute to the alleviation of CKD occurrence [39]. Thus, further studies should consider the nutritional status of participants as a potential confounding variable. Moreover, because our study was limited to elderly populations, our results cannot be generalized to all age groups. According to previous studies, edentulous participants may have a higher risk for CKD $[14,15]$, as such, it may be necessary to consider edentates separately. Lastly, this cross-sectional study does not have the ability to identify causal relationships; further well-designed longitudinal studies are needed to determine the biological mechanisms for the presented association.

Notwithstanding, our study has major strengths. First, we evaluated the definition and classification system for CKD and applied them through GFR and albuminuria categories as proposed by KDIGO 2012. An updated 
KDIGO guideline provides relatively easy and accurate methods for early detection of CKD, compared to the CKD-EPI (chronic kidney disease-epidemiology collaboration) equation and MDRD study equation [40]. Second, the number of teeth and oral health status in the entire oral cavity were objectively examined by dentists. Third, general health examination and laboratory analyses were documented by professional examiners. Fourth, the results were acquired from elder participants who represent the Korean population from the KNHANES data. These results provide the first evidence linking the number of existing permanent teeth and CKD among older adults. Thus, medical and dental professionals should be concerned with the association between the number of teeth, as an endpoint of oral health, and CKD.

\section{KEY MESSAGE}

1. The number of existing permanent teeth is associated with chronic kidney disease in Korean elderly population.

2. The lower number of existing permanent teeth was high in those with chronic kidney disease. The link was highlighted in females aged 75 years over.

3. Medical and dental professionals should be concerned with the link between the number of teeth and chronic kidney disease at old age.

\section{Conflict of interest}

No potential conflict of interest relevant to this article was reported.

\section{REFERENCES}

1. Go AS, Chertow GM, Fan D, McCulloch CE, Hsu CY. Chronic kidney disease and the risks of death, cardiovascular events, and hospitalization. N Engl J Med 2004;351:1296-1305.

2. Levin A, Stevens PE. Summary of KDIGO 2012 CKD guideline: behind the scenes, need for guidance, and a framework for moving forward. Kidney Int 2014;85:49-61.

3. Ji E, Kim YS. Prevalence of chronic kidney disease defined by using CKD-EPI equation and albumin-to-cre- atinine ratio in the Korean adult population. Korean $\mathrm{J}$ Intern Med 2016;31:1120-1130.

4. Kinane DF. Causation and pathogenesis of periodontal disease. Periodontol 2000 2001;25:8-20.

5. Jepsen S, Stadlinger B, Terheyden H, Sanz M. Science transfer: oral health and general health: the links between periodontitis, atherosclerosis and diabetes. J Clin Periodontol 2015;42:1071-1073.

6. Han DH, Lim S, Paek D, Kim HD. Periodontitis could be related factors on metabolic syndrome among Koreans: a case-control study. J Clin Periodontol 2012;39:30-37.

7. Aida J, Ando Y, Akhter R, Aoyama H, Masui M, Morita M. Reasons for permanent tooth extractions in Japan. J Epidemiol 2006;16:214-219.

8. Kassebaum NJ, Bernabe E, Dahiya M, Bhandari B, Murray CJ, Marcenes W. Global burden of severe tooth loss: a systematic review and meta-analysis. J Dent Res 2014;93:20S-28S.

9. Korea Centers for Disease Control and Prevention. The Fourth Korea National Health and Nutrition Examination Survey. Cheongju: Korea Centers for Disease Control and Prevention, 2014.

10. Chambrone L, Foz AM, Guglielmetti MR, et al. Periodontitis and chronic kidney disease: a systematic review of the association of diseases and the effect of periodontal treatment on estimated glomerular filtration rate. J Clin Periodontol 2013;40:443-456.

11. Ricardo AC, Athavale A, Chen J, et al. Periodontal disease, chronic kidney disease and mortality: results from the third National Health and Nutrition Examination Survey. BMC Nephrol 2015;16:97.

12. Iwasaki M, Taylor GW, Nesse W, Vissink A, Yoshihara A, Miyazaki H. Periodontal disease and decreased kidney function in Japanese elderly. Am J Kidney Dis 2012;59:202209.

13. Kshirsagar AV, Moss KL, Elter JR, Beck JD, Offenbacher S, Falk RJ. Periodontal disease is associated with renal insufficiency in the Atherosclerosis Risk In Communities (ARIC) study. Am J Kidney Dis 2005;45:650-657.

14. Fisher MA, Taylor GW, Papapanou PN, Rahman M, Debanne SM. Clinical and serologic markers of periodontal infection and chronic kidney disease. J Periodontol 2008;79:1670-1678.

15. Fisher MA, Taylor GW, Shelton BJ, et al. Periodontal disease and other nontraditional risk factors for CKD. Am J Kidney Dis 2008;51:45-52. 
16. Grubbs V, Plantinga LC, Crews DC, et al. Vulnerable populations and the association between periodontal and chronic kidney disease. Clin J Am Soc Nephrol 2011;6:711717 .

17. Brotto RS, Vendramini RC, Brunetti IL, Marcantonio RA, Ramos AP, Pepato MT. Lack of correlation between periodontitis and renal dysfunction in systemically healthy patients. Eur J Dent 2011;5:8-18.

18. Iwasaki M, Taylor GW, Manz MC, et al. Serum antibody to Porphyromonas gingivalis in chronic kidney disease. J Dent Res 2012;91:828-833.

19. Ioannidou E, Hall Y, Swede H, Himmelfarb J. Periodontitis associated with chronic kidney disease among Mexican Americans. J Public Health Dent 2013;73:112-119.

20. Sharma P, Dietrich T, Sidhu A, et al. The periodontal health component of the Renal Impairment In Secondary Care (RIISC) cohort study: a description of the rationale, methodology and initial baseline results. J Clin Periodontol 2014;41:653-661.

21. Liu K, Liu Q, Chen W, et al. Prevalence and risk factors of CKD in Chinese patients with periodontal disease. PLoS One 2013;8:e70767.

22. Brito F, Almeida S, Figueredo CM, Bregman R, Suassuna JH, Fischer RG. Extent and severity of chronic periodontitis in chronic kidney disease patients. J Periodontal Res 2012;47:426-430.

23. Han SS, Shin N, Lee SM, Lee H, Kim DK, Kim YS. Correlation between periodontitis and chronic kidney disease in Korean adults. Kidney Res Clin Pract 2013;32:164170.

24. Levey AS, Stevens LA, Schmid CH, et al. A new equation to estimate glomerular filtration rate. Ann Intern Med 2009;150:604-612.

25. Levey AS, Coresh J, Greene T, et al. Expressing the modification of diet in renal disease study equation for estimating glomerular filtration rate with standardized serum creatinine values. Clin Chem 2007;53:766-772.

26. Jeong TD, Lee W, Chun S, et al. Comparison of the MDRD study and CKD-EPI equations for the estimation of the glomerular filtration rate in the Korean general population: the fifth Korea National Health and Nutrition Examination Survey (KNHANES V-1), 2010. Kidney Blood Press Res 2013;37:443-450.

27. Park JI, Baek H, Jung HH. Prevalence of chronic kidney disease in Korea: the Korean National Health and Nutri- tional Examination Survey 2011-2013. J Korean Med Sci 2016;31:915-923.

28. Han DH, Khang YH. Lifecourse socioeconomic position indicators and tooth loss in Korean adults. Community Dent Oral Epidemiol 2017;45:74-83.

29. Han DH, Khang YH, Lee HJ. Association between adult height and tooth loss in a representative sample of Koreans. Community Dent Oral Epidemiol 2015;43:479-488.

30. Artese HP, Sousa CO, Luiz RR, Sansone C, Torres MC. Effect of non-surgical periodontal treatment on chronic kidney disease patients. Braz Oral Res 2010;24:449-454.

31. Sharma P, Dietrich T, Ferro CJ, Cockwell P, Chapple IL. Association between periodontitis and mortality in stages 3-5 chronic kidney disease: NHANES III and linked mortality study. J Clin Periodontol 2016;43:104-113.

32. Iwasaki M, Kimura Y, Yoshihara A, et al. Association between dental status and food diversity among older Japanese. Community Dent Health 2015;32:104-110.

33. Lee IC, Yang YH, Ho PS, Lee IC. Chewing ability, nutritional status and quality of life. J Oral Rehabil 2014;41:7986.

34. Hung HC, Joshipura KJ, Jiang R, et al. Fruit and vegetable intake and risk of major chronic disease. J Natl Cancer Inst 2004;96:1577-1584.

35. Schulze MB, Liu S, Rimm EB, Manson JE, Willett WC, $\mathrm{Hu}$ FB. Glycemic index, glycemic load, and dietary fiber intake and incidence of type 2 diabetes in younger and middle-aged women. Am J Clin Nutr 2004;80:348-356.

36. Conlin PR, Chow D, Miller ER 3rd, et al. The effect of dietary patterns on blood pressure control in hypertensive patients: results from the Dietary Approaches to Stop Hypertension (DASH) trial. Am J Hypertens 2000;13:949-955.

37. Kressin NR, Boehmer U, Nunn ME, Spiro A 3rd. Increased preventive practices lead to greater tooth retention. J Dent Res 2003;82:223-227.

38. Ioannidou E, Swede H, Fares G, Himmelfarb J. Tooth loss strongly associates with malnutrition in chronic kidney disease. J Periodontol 2014;85:899-907.

39. Anderson CA, Nguyen HA, Rifkin DE. Nutrition interventions in chronic kidney disease. Med Clin North Am 2016;100:1265-1283.

40. Levey AS, Becker C, Inker LA. Glomerular filtration rate and albuminuria for detection and staging of acute and chronic kidney disease in adults: a systematic review. JAMA 2015;313:837-846. 